\title{
Pathologic Active mTOR Mutation in Brain Malformation with Intractable Epilepsy Leads to Cell-Autonomous Migration Delay
}

Sae Hanai, ${ }^{\dagger \dagger}$ Sayuri Sukigara, ${ }^{* \dagger}$ Hongmei Dai, ${ }^{\dagger}$ Tomoo Owa, ${ }^{\ddagger}$ Shin-ichi Horike, ${ }^{\S}$ Taisuke Otsuki, ${ }^{*}$ Takashi Saito, ${ }^{*}$ Eiji Nakagawa, ${ }^{* \|}$ Naoki Ikegaya, ${ }^{* \pi}$ Takanobu Kaido, ${ }^{*}$ Noriko Sato, ${ }^{* * *}$ Akio Takahashi, ${ }^{* \pi}$ Kenji Sugai, ${ }^{*} \|$ Yuko Saito, ${ }^{*}{ }^{* \dagger}$ Masayuki Sasaki, ${ }^{* \|}$ Mikio Hoshino, ${ }^{* \ddagger}$ Yu-ichi Goto, ${ }^{* \dagger}$ Schuichi Koizumi, ${ }^{ \pm \star}$ and Masayuki Itoh ${ }^{* \dagger}$

From the Epilepsy Center,* National Center of Neurology and Psychiatry, the Departments of Mental Retardation and Birth Defect Research ${ }^{\dagger}$ and Biochemistry and Cellular Biology, ${ }^{\ddagger}$ National Institute of Neuroscience, Kodaira; the Division of Functional Genomics, ${ }^{\S}$ Advanced Science Research Center Kanazawa University, Kanazawa; the Departments of Neurosurgery, ${ }^{9}$ Child Neurology, ${ }^{\|}$Radiology, $* *$ and Laboratory Medicine ${ }^{\dagger \dagger}$ Hospital of National

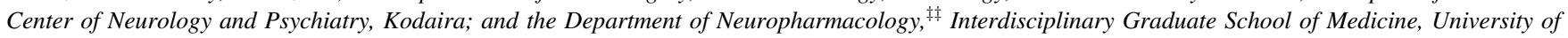
Yamanashi, Chuo, Japan

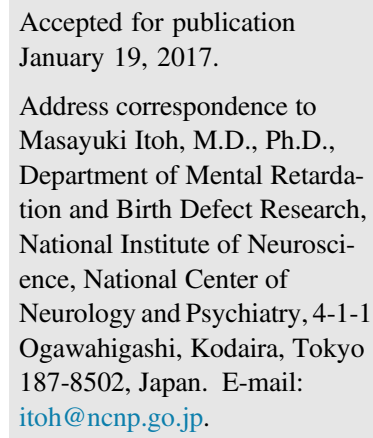

\begin{abstract}
The activation of phosphatidylinositol 3-kinase-AKTs-mammalian target of rapamycin cell signaling pathway leads to cell overgrowth and abnormal migration and results in various types of cortical malformations, such as hemimegalencephaly (HME), focal cortical dysplasia, and tuberous sclerosis complex. However, the pathomechanism underlying abnormal cell migration remains unknown. With the use of fetal mouse brain, we performed causative gene analysis of the resected brain tissues from a patient with HME and investigated the pathogenesis. We obtained a novel somatic mutation of the MTOR gene, having approximately $11 \%$ and $7 \%$ mutation frequency in the resected brain tissues. Moreover, we revealed that the MTOR mutation resulted in hyperphosphorylation of its downstream molecules, S6 and 4E-binding protein 1, and delayed cell migration on the radial glial fiber and did not affect other cells. We suspect cell-autonomous migration arrest on the radial glial foot by the active MTOR mutation and offer potential explanations for why this may lead to cortical malformations such as HME. (Am J Pathol 2017, 187: 1177-1185; http://dx.doi.org/10.1016/ j.ajpath.2017.01.015)
\end{abstract}

Brain malformations with intractable epilepsy may occur in isolation or in diverse megalencephaly syndromes, including the wide spectrum from microdysplastic cortex to huge megalencephaly. ${ }^{1}$ Among them, hemimegalencephaly (HME) and focal cortical dysplasia (FCD) are relatively frequent in childhood epilepsy and often can be treated by surgery. ${ }^{2}$ The histopathologic process in HME and FCD brains is characterized by the features of cortical dyslamination, dysmorphic immature neurons, and neuronal heterotopia, indicating primary defects in neuroglial differentiation, migration, and cellular growth. Interestingly, this pathologic disorder shares similarities with tuberous sclerosis complex (TSC), having multiple cortical tubers and showing infantile spasm and various mental retardations. Of TSC causative genes, TSC1 or TSC2 also control the mammalian target of rapamycin
(mTOR) activity. mTOR is a large molecule, encoded by the MTOR gene (1p36), and its structure consists of HEAT repeats (interaction domain with regulatory-associated protein of mTOR or rapamycin-insensitive companion of mTOR),

\footnotetext{
Supported by the National Center of Neurology and Psychiatry Intramural Research grants $27-7$ (E.N., M.I.) and 28-4 (M.H., M.I.) for Neurological and Psychiatric Disorders, the Japan Society for the Promotion of Science grant-in-aid 25670486 for Challenging Exploratory Research (M.I.), and the Kawano Masanori Memorial Public Interest Incorporated Foundation for Promotion of Pediatrics grant-in-aid (M.I.)

Disclosures: None declared.

Current address of T.Ot., Epilepsy Hospital Bethel Japan, Sendai, Japan; of T.K., National Hospital Organization Nara Medical Center, Nara, Japan; of A.T., National Hospital Organization Shibukawa Medical Center, Shibukawa, Japan.
} 
FRAP-ATM-TTRAP (FAT; protein-protein interaction domain), FRB (rapamycin binding domain), kinase domain (interaction with mammalian lethal with SEC13 protein 8), and catalytic active domain in C-terminus and interaction sites of regulatory-associated protein of mTOR, rapamycin-insensitive companion of mTOR, and mammalian lethal with SEC13 protein $8 .{ }^{3}$ By input from upstream regulatory proteins of growth factors, ATP, and so on, MTOR makes phosphorylation of downstream proteins to cell growth, conservancy, and survival. MTOR also leads to the phosphorylation of S6 kinase 1 and eukaryotic initiation factor 4E-binding protein 1 (4EBP1), direct regulators of translation initiation, and results to induce translation pathway and increase in cell size, as well as migratory and invasive behavior of cells. ${ }^{4}$ Clinically, activated mTOR is well known for forming various megalencephalies and tumors. ${ }^{5}$ Mutations of TSC1 or TSC2 result in the progressive phosphorylation of mTOR, showing hyperactivation of the mTOR pathway, which may be a common mechanism underlying the disorders. Recently, many causative genes of HME and FCD were described in somatic or germline mutations. AKT1 was the first gene causally linked to HME. ${ }^{6}$ Moreover, several HMEs are caused by de novo mutations in PIK3CA, PIK3R2, AKT3, and MTOR ${ }^{7-9}$ FCD type IIa was linked to a somatic mutation in PIK $3 C A .{ }^{10}$ Most of the gene products belong to the major molecules of phosphatidylinositol 3-kinase (PI3K)AKTs-mTOR cell signaling pathway and conclusively upregulate mTOR signaling, resulting in cell overgrowth and abnormal migration through hyperactive phosphorylated proteins. ${ }^{11}$ However, Mtor-null mice revealed embryonic lethality at the early stage, whereas heterozygous mice showed normal development. ${ }^{12,13}$ In addition, mTOR splicing defect mutant embryos lack a telencephalon and die at mid-gestation. ${ }^{14}$ Moreover, mTOR inactivation caused microcephaly and disruption of peripheral nerve growth in mice. ${ }^{15,16}$ Taken together, the well-controlled PI3K-AKTs-mTOR signaling pathway is important in normal development. In megalencephalic brains of HME, FCD, and TSC, we previously described immaturity and mis-positioning of cortical neurons. ${ }^{17-20}$ However, an important problem remains to be addressed, concerning the mechanism of neuronal migration disruption.

Here, we discovered a novel somatic MTOR mutation in a HME patient. Furthermore, we characterized the cellautonomous arrest of neuronal migration on the radial glial foot by the active mTOR mutation. We demonstrate that the active mTOR mutation affects radial neuronal migration and cell growth in the embryonic period and speculate that the result forms HME with intractable epilepsy.

\section{Materials and Methods}

\section{Patient with HME and Neuropathology}

Our patient, who was the first female from nonconsanguineous parents without any neurologic history, exhibited daily cluster-formed spasms with a suppression-burst pattern on electroencephalograph (Figure 1G) from 1 month old and demonstrated left HME by magnetic resonance imaging (Figure 1A), and the lesion exhibited hyperperfusion (Figure 1C) and hypermetabolism (Figure 1E). At 3 months of age, operation of the left hemisphere disconnection (Figure 1B) was performed for the treatment of her intractable epilepsy and improvement of electroencephalograph (Figure 1H). For pathologic diagnosis and exploration of causative genes, fresh-frozen and formalin-fixed tissues from two regions (cortex and white matter) (Figure 1A) were stored. The pathologic examination revealed FCD type IIa, characterized by many dysmorphic neurons with a unilayer pattern of the cortex and gliosis (Figure 1, I-L). After surgery, the contralateral perfusion and metabolism were recovered (Figure 1, D and F). Then, she was seizure free and had minimal electroencephalograph abnormality for 4 years (Figure 1H).

The study was approved by the Ethical Committee of the National Center of Neurology and Psychiatry, and informed consent from her parents for genetic analysis was obtained.

\section{Explanation of Causative Gene Analysis}

Genomic DNA from frozen brain and peripheral blood were extracted using a DNeasy Blood and Tissue Kit (Qiagen, Venlo, the Netherlands). For the genetic variation study, target sequencing was conducted in the genomic DNAs on an Ion PGM System (Life Technologies, Carlsbad, CA). The targeted genes included 17 major genes of the PI3K-AKTs-mTOR signaling pathway: IRS1, PIK3CA, PIK3R1, PDK1, AKT1, AKT2, AKT3, PTEN, INPPRL1, FOXO1, FOXO3, FOXO4, AGAP2, TSC1, TSC2, RHEB, and MTOR. Targeted exon enrichment was performed using the GeneRead DNA panel. Enrichment was accomplished using 20 to 25 PCR cycles. Library construction was accomplished using Ion AmpliSeq Designer and Library Kit 2.0 (Life Technologies). The sequence data were obtained after subtraction from the pathologic brain-derived genome to the blood-derived genome. The sequence traces were mapped to the human reference genome (hg19) with the genome browser at the University of California Santa Cruz (http://genome.ucsc.edu, last accessed January 10, 2017). The mean depth of coverage was 100 reads, with approximately $95 \%$ of all coding exons being covered by 10 or more reads. Of all variants within exons and 20-bp intronic regions from the exon-intron boundaries, those registered in dbSNP135 (http://www.ncbi.nlm.nih.gov/projects/SNP, last accessed January 10, 2017), 1000 Genomes (http:// www.ncbi.nlm.nih.gov/variation/tools/1000genomes, last accessed January 10, 2017), and the Catalog of Somatic Mutations in Cancer (http://cancer.sanger.ac.uk/cosmic, last accessed January 10, 2017) were removed. As estimated, somatic or heterozygous variant candidates were identified. Then, searches were conducted for mutations in the regions 

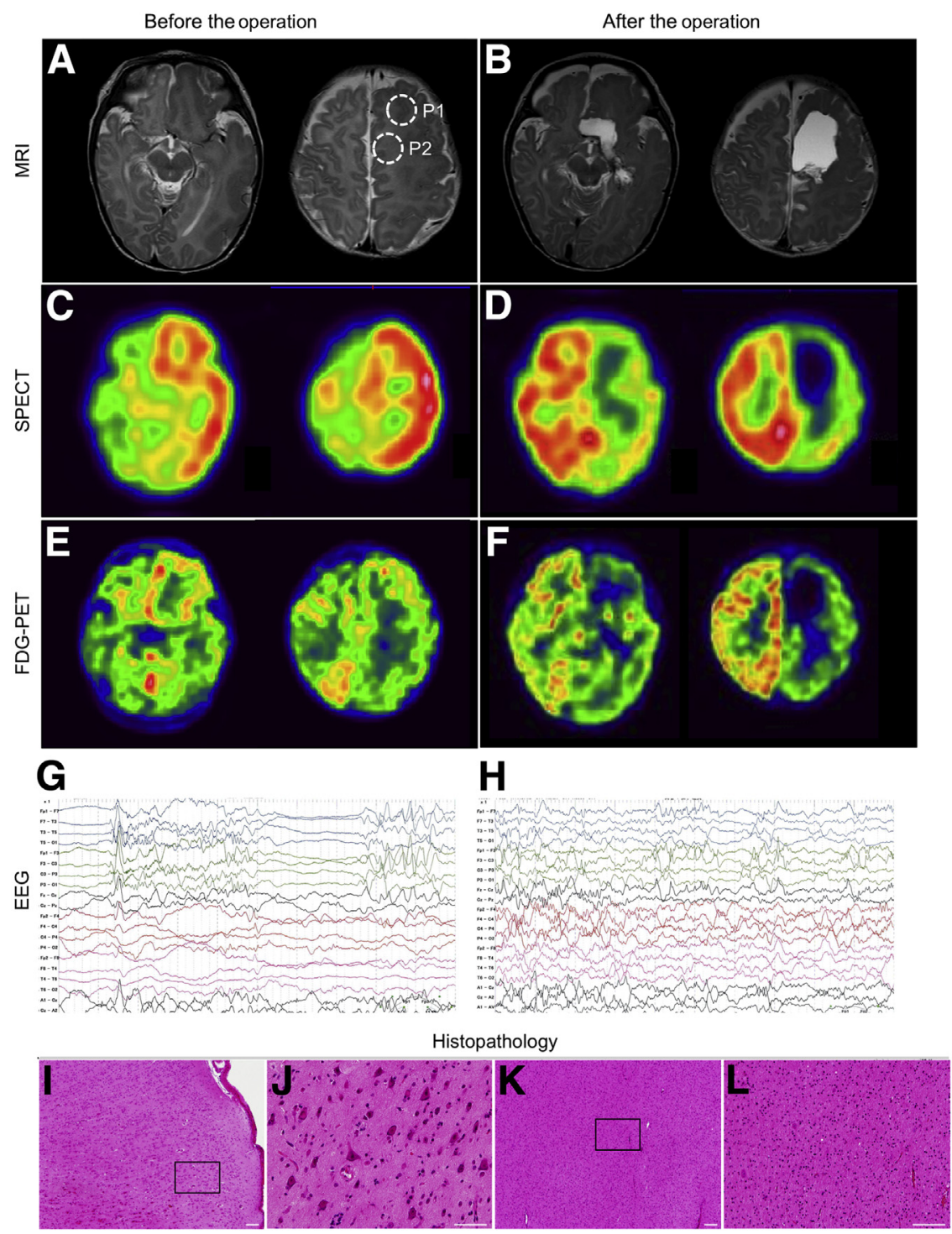

Figure 1 Characteristic imaging, electroencephalograph (EEG), and histopathology of a patient. A: Axial T2-weighted image acquisition performed at age 3 months showing blurring of the gray matter, simplified sulcation, and hypointense white matter in the left frontal and central regions consistent with extensive cortex. Circle P1 and P2 are removed tissues by the operation and performed neuropathologic and genetic examinations of $\mathbf{I}-\mathbf{L}$ and Figure 2. B: Axial T2-weighted images performed 2 weeks after left hemispherectomy demonstrate the resected areas. C: Interictal SPECT images show hyperperfusion of the left hemisphere and contralateral hypoperfusion. D: SPECT images after the operation show hypoperfusion of the left hemisphere and recovery of contralateral perfusion. E: Interictal fluorodeoxyglucose-PET images consistent with hypermetabolism of the left hemisphere. F: PET images after the operation show remaining hypometabolism of the left hemisphere and recovery of contralateral metabolism. G: EEG at age 2 months shows a typical suppression-burst pattern before the operation. H: EEG after the operation shows the suppressionburst pattern has disappeared. I and J: The resected tissue from P1 in $\mathbf{A}$ demonstrates hypercellularity and a unilayer pattern (H\&E staining) and many dysmorphic neurons. Boxed area in $\mathbf{I}$ is shown at higher magnification in $\mathbf{J}$. $\mathbf{K}$ and $\mathbf{L}$ : The resected tissue from P2 in $\mathbf{A}$ shows marked gliosis (H\&E staining) and scattered heterotopic neurons Boxed area in $\mathbf{K}$ is shown at higher magnification in L. Original magnification: $\times 4(\mathbf{I}$ and $\mathbf{K}) ; \times 10$ (J and L). Scale bars: $200 \mu \mathrm{m}$ (I and $\mathbf{K}$ ); $100 \mu \mathrm{m}$ (J and L). FDG, fluorodeoxyglucose; H\&E, hematoxylin and eosin; MRI, magnetic resonance imaging; $\mathrm{PET}$, positron emission tomography; $\mathrm{P} 1$, cortex; P2, white matter; SPECT, single-photon emission computed tomography. of exons and splicing sites using CLC Genomic Workbench (CLC Bio Japan, Tokyo, Japan), focusing on $>10 \%$ allele variation appearance with a coverage $>30$ as definitive mutations. From the result, the candidate variant was in only one gene of MTOR. To confirm the mutations, direct sequencing was performed by the Sanger method with an ABI PRISM 3130 genetic analyzer (Applied Biosystems, Foster City, CA). The primer set for Sanger sequencing was a forward sequence of $5^{\prime}$-CTGTGATAGGTGAAGTGTCATGC- $3^{\prime}$ and a reverse sequence of 5'-AGCCACACATGCCATCATTCTA- $3^{\prime}$. Next, to identify fractions of candidate variation, pyrosequencing analysis was performed with the PyroMark Q24 advanced system (Qiagen). All of the primers were designed with the PyroMark Assay Design Software version 2.0 (Qiagen) with a forward primer of $5^{\prime}$ GAGAAACTGCACGAGTGGG-3', a reverse primer of $5^{\prime}$ TCGTCCTTGTTGGTGTCCA-3' ${ }^{\prime}$, and a sequencing primer of $5^{\prime}$-CACGAGTGGGAGGAT-3'. The pyrosequencing running protocol was planned and the plate was set up. Then, each DNA sample was assayed at target sequence analysis three times, and the sequence traces were analyzed using PyroMark Q24 advanced software version 3.00 (Qiagen).

Immunohistochemistry and Immunoblotting Analysis of the HME Patient's Brain

After diagnosed pathology of the resected tissues, paraffinembedded samples were used for immunohistochemistry and frozen tissues of the same resected regions for immunoblotting analysis. Immunohistochemistry was performed as previously described. ${ }^{21}$ The primary antibodies of MTOR [1/200 of dilution with $1 \%$ bovine serum albumin in phosphate-buffered saline (PBS); Abnova, 
Taipei, Taiwan], S6 (1/100; Cell Signaling Technology, Danvers, MA), phosphorylated (Ser235/236)-S6 (1/100; Cell Signaling Technology), 4EBP1 (1/100; Abcam, Cambridge, UK), and phosphorylated (Thr37/46)-4EBP1 (1/200; Cell Signaling Technology) were incubated with the deparaffinized sections at $4^{\circ} \mathrm{C}$ overnight. For visualization, Alexa Fluor 488-conjugated donkey anti-mouse IgG (Invitrogen, Carlsbad, CA) and Alexa Fluor 594conjugated chicken anti-rabbit IgG (Invitrogen) secondary antibodies were used, and the slides were mounted with DAPI (Vector Laboratories, Burlingame, CA) for labeling nuclei. Then, the reactivated sections were observed and photographed using a fluorescent microscope (U-TB190; Olympus, Tokyo, Japan).

Protein from prepared tissues was extracted according to a previously described procedure. ${ }^{11}$ The tissues selected for analysis were adjacent to the areas with pathologic findings and controls, and $50 \mu \mathrm{g}$ of the extracted proteins was loaded on each lane. After transfer to a polyvinylidine difluoride membrane, each immunoreacted band was detected using an enhanced chemiluminescence system (Amersham Pharmacia Biotech, Buckinghamshire, UK) according to the manufacturer's instructions. As a reference, $\beta$-actin was detected using a specific antibody (Sigma-Aldrich, St. Louis, MO). The expression levels of detected bands were measured and calculated by ImageQuant TL 7.0 (GE Healthcare, Buckinghamshire, UK) and compared with those of each sample.

\section{Preparation of Human MTOR cDNA Vectors}

A human MTOR cDNA-conjugated pCMV6 vector was obtained from OriGene (RC220457; Rockville, MD). A pCAG in promoter was converted, and MTOR wild-type cDNA or mutated cDNA was inserted into multiple cloning sites of the vector. After confirming the cDNA sequences (https://www.ncbi.nlm.nih.gov/nuccore; accession number NM_004958.3), the sequences and a non-cDNA vector (empty vector; mock) was used to transfect into culture cells and the ventricular space of fetal brains.

\section{Assay for MTOR Activation in Cultured Cells}

Immunocytochemistry and immunoblotting analyses were performed with vector-transfected HeLa cells. The transfection protocol used $2.0 \mu \mathrm{g} / \mu \mathrm{L}$ of each MTOR cDNAvector with cotransfection of $0.5 \mu \mathrm{g} / \mu \mathrm{L}$ enhanced green fluorescent protein (EGFP) by Lipofectamine 3000 (Invitrogen). At 24 hours after transfection, a transfection efficiency of approximately $50 \%$ was confirmed by counting the EGFP-positive cells. The cells were then washed with PBS and lyzed on ice using a freshly prepared ice-cold cell lysis buffer containing $1 \% \mathrm{NP}-40$ in $150 \mathrm{mmol} / \mathrm{L} \mathrm{NaCl}$ and $50 \mathrm{mmol} / \mathrm{L}$ Tris $(\mathrm{pH} 7.5), 2 \mathrm{mmol} / \mathrm{L}$ EDTA containing phosphatase inhibitors (Roche Diagnostics, Basel, Switzerland) and protease inhibitors (Sigma-Aldrich). Cell lysates were collected, incubated on $4^{\circ} \mathrm{C}$ for 1 hour, and centrifuged at $13,000 \mathrm{rpm}$ for 30 minutes at $4^{\circ} \mathrm{C}$. The supernatant fluids were collected, and protein concentrations were measured using the Pierce BCA protein assay kit (Thermo Fisher Scientific, Waltham, MA). This cell lysate was used for immunoblotting analysis.

\section{In Utero Electroporation and Quantification}

Pregnant ICR mice were purchased from CREA Japan Inc. (Tokyo, Japan). In utero electroporation was performed as described previously. ${ }^{22}$ Briefly, pregnant mice carrying embryonic day (E)14.5 embryos were anesthetized. One microliter of $2.0 \mu \mathrm{g} / \mu \mathrm{L}$ vectored DNA in water containing Fast Green (Sigma-Aldrich) was injected into the lateral ventricle of embryonic brains. Holding the embryo in utero with a forceps-type electrode (Nepa Gene Co. Ltd., Chiba, Japan), 50 milliseconds of $35-\mathrm{V}$ electronic discharge were delivered five times at intervals of 450 milliseconds with the electroporator (CUY21EDIT; Nepa Gene Co. Ltd.). After the embryos were
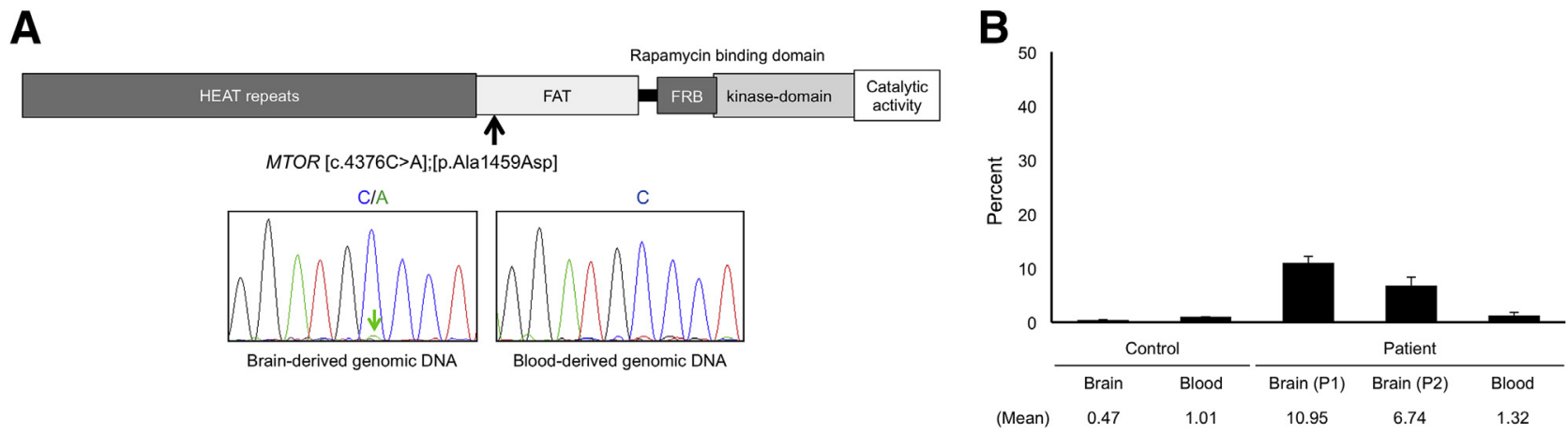

Figure 2 Localization of c.4376C $>$ A, p.Ala1459Asp mutation in MTOR gene and its allelic frequency in the pathologic lesions. A: The c.4376C $>$ A, p.Ala1459Asp mutation locates in FAT domain of MTOR gene. Sanger sequencing shows the mutation only in the brain-derived genomic DNA. However, its signal is weak (green arrow). B: Pyrosequencing reveals approximately $11 \%$ mutation allelic frequency in the resected tissue Brain (P1) (Figure 1) and approximately $7 \%$ in the Brain (P2) (Figure 1) but $\leq 1 \%$ in the control samples. Data are expressed as means \pm SEM. $n=3$ control samples. FAT, FRAP-ATM-TTRAP; FRB, rapamycin binding domain; P1, cortex; P2, white matter. 
grown for 4 days (E18.5), the brains were removed, fixed with $4 \%$ paraformaldehyde in phosphate buffer, and cut coronally into $18-\mu \mathrm{m}$ slices with a cryostat (CM3050S; Leica Microsystems $\mathrm{GmbH}$, Wetzlar, Germany). Sections were stained by DAPI, and the fluorescence images of brains were captured by a U-TB190 (Olympus) or a LSM780 laser scanning confocal microscope (Carl Zeiss AG, Oberkochen, Germany).

After rescanning the figures into the computer, the distribution and cell size [soma and nuclear size $\left(\mu \mathrm{m}^{2}\right)$ ] of cortical neurons were quantified using ImageJ software version 1.48 (NIH, Bethesda, MD; https://imagej.nih.gov/ij). EGFP-positive cells were counted, and the distribution ratio of each cortical region (layers I and II/III, layer IV, and lower layers $\mathrm{V}$ and VI [chicken ovalbumin upstream promoter transcription factor-interacting proteins 2 (Ctip2)labeled cell localization]) was calculated. After a cell size ratio [soma area $\left(\mu \mathrm{m}^{2}\right) /$ nuclear area $\left(\mu \mathrm{m}^{2}\right)$ ] in each cell was obtained from EGFP-positive area by DAPI-positive area, the data were analyzed and compared with data from each vector-transfected brain group.

Moreover, immunohistochemistry of serial frozen sections of the somatosensory cortex was performed. After washing with PBS and blocking with $2 \%$ bovine serum albumin and $0.1 \%$ Triton $\mathrm{X}$ with PBS, the sections were incubated overnight at $4{ }^{\circ} \mathrm{C}$ with the primary antibodies of Ctip2 (1/50; Abcam), forkhead box protein P1 (FOXP1; 1/ 200; Abcam), Vimentin (1/50; Santa Cruz Biotechnology, Santa Cruz, CA). The sections were sequentially incubated with the Alexa Fluor-conjugated secondary antibodies and mounted with DAPI. Fluorescent sections were observed on a microscope or a confocal microscope.

\section{Statistical Analysis}

The relation between two treated groups (wild-type MTOR and mutated MTOR vector-transfected cell groups) and one
A

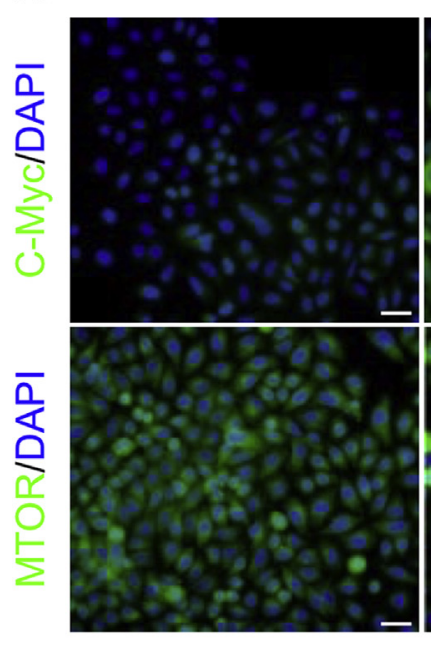
MTOR-WT

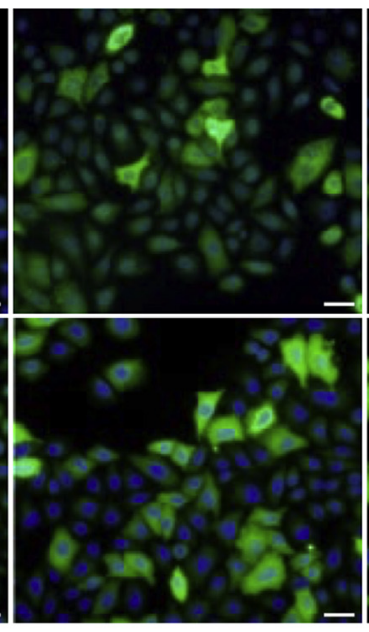

B

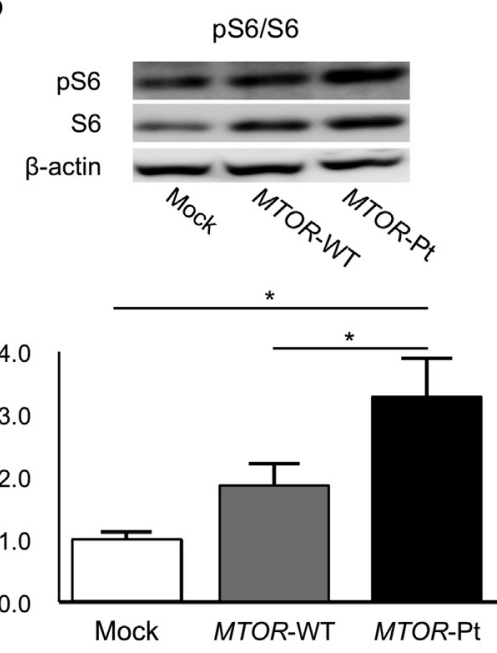

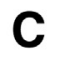
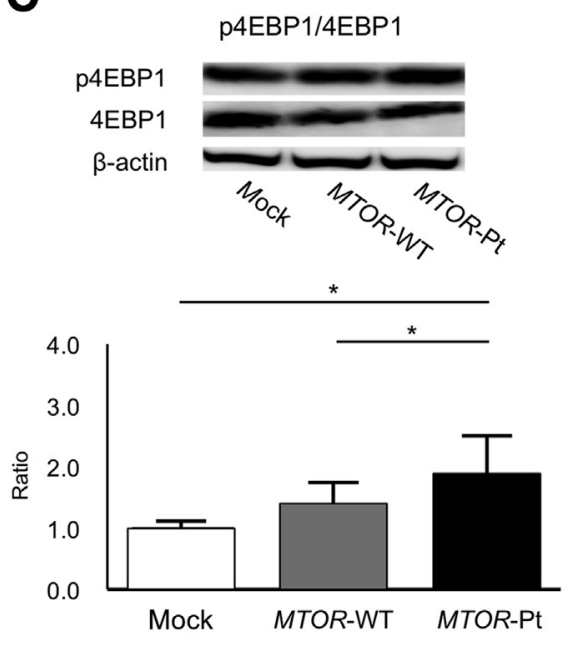

Figure 3 Cellular effects of mutated MTOR on HeLa cells. A: MTOR-Pt cells (green) demonstrate enlargement and multinucleated cells. B and C: Comparative expression of the three transfected cells reveals significant higher levels of $\mathrm{pS} 6 / \mathrm{S} 6$ (B) and $\mathrm{p} 4 \mathrm{EBP} 1 / 4 \mathrm{EBP} 1$ (C) in MTOR-Pt than in MTORWT and mock. Data are expressed as means \pm SEM. $n=6$ MTOR-Pt cells (B and C); $n=3$ MTOR-WT and mock cells (B and $\mathbf{C}$ ). ${ }^{*} P<0.05$. Scale bars $=30 \mu \mathrm{m}$. MTOR-Pt, patient's mutated mTOR transfected cells; MTOR-WT, wild-type $M T O R$ transfected cells; p4EBP1, phosphorylated $4 \mathrm{E}$ binding protein 1 . 

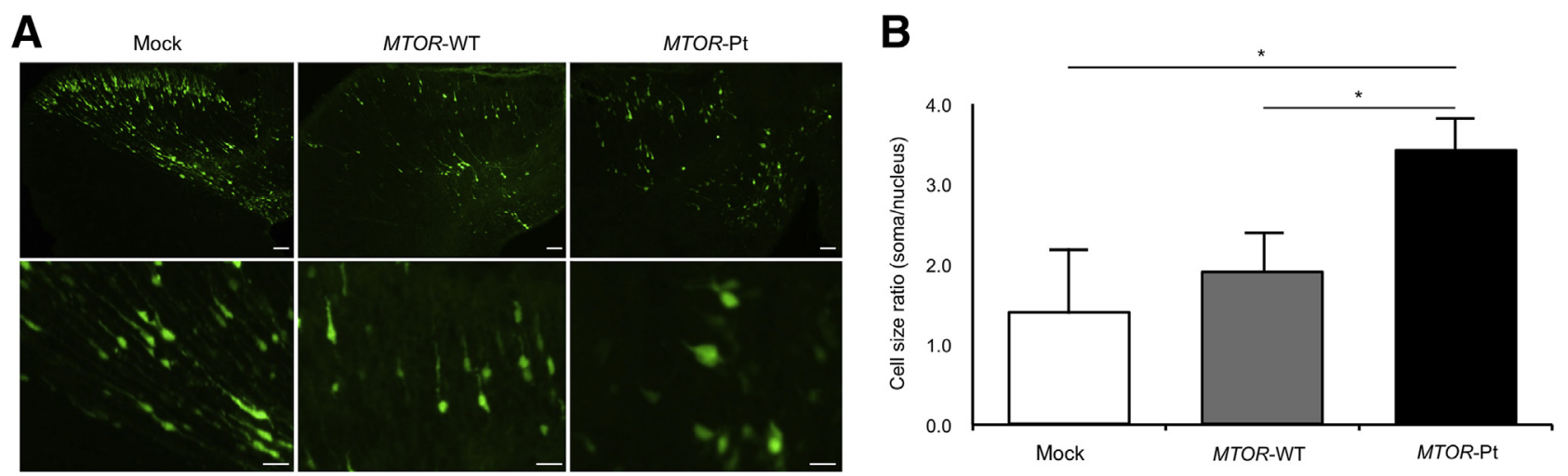

Figure 4 Comparison of cell size between mock, MTOR-WT, and MTOR-Pt. A: Three transfected cells distributed in the neocortex. Mock cells show pyramidal shape and well-formed dendrites. MTOR-WT cells also show pyramidal shape but thinning dendrites. MTOR-Pt cells reveal large soma and short dendrites. B: From the ratio of soma/nucleus areas, MTOR-Pt cell size is larger than those of mock and MTOR-WT. Data are expressed as means \pm SEM. $n=3$ MTOR-Pt cells (B); $n=3$ mock cells (B); $n=4$ MTOR-WT (B). ${ }^{*} P<0.05$. Scale bars $=20 \mu \mathrm{m}$. MTOR-Pt, patient's mutated mTOR transfected cells; MTOR-WT, wild-type $m$ TOR transfected cells.

control group (mock transfected cell group) was evaluated, using Dunnett's test for many-to-one comparison analysis ${ }^{23}$ with SPSS statistical software version 24.0 (SPSS Inc., Chicago, IL) with a significance level of $P<0.05$.

\section{Results}

\section{Causative Somatic Mutation of MTOR Gene}

After genetic analyses of a patient, we identified a brainspecific somatic missense mutation of c.4376C $>$ A, p.Ala1459Asp in the MTOR gene and a mutated allele frequency of $15.63 \%$ by the target sequencing. A pyrosequencing analysis for the mutation resulted in approximately $10 \%$ variant allele appearance (Figure 2). Interestingly, the white matter tissue (Figure 1A) had a relatively low frequency. Our patient's mutation position in MTOR gene, located into the FAT kinase domain, is a novel finding, but its near-position mutations have already been reported pathologically. ${ }^{24,25}$

\section{MTOR Activation by the Identified Mutation}

At first, we confirmed MTOR activation in the patient's brain. The pS6 and p4EBP1 were heavily labeled in TSC and HME brains examined but not in the control (Supplemental Figures S1 and S2). Those immunoreactivities were observed in the soma and cellular processes of dysplastic neurons. The pS6 or p4EBP1 expression levels in the patient's brain demonstrated dispersed colocalization with MTOR and higher levels than those of control (Supplemental Figures S1 and S2). It was suggested that the epileptogenic pathology exhibited significant up-regulation of MTOR protein kinase activity.

In addition, MTOR-mutant HeLa cells showed larger cell size and higher pS6 and p4EBP1 expression levels (Figure 3). The expression levels in normal MTOR-transfected cells also exhibited relatively higher expression than in control cells but less than significantly in MTOR-mutant cells. The in vitro study indicated that a high dose of MTOR
A

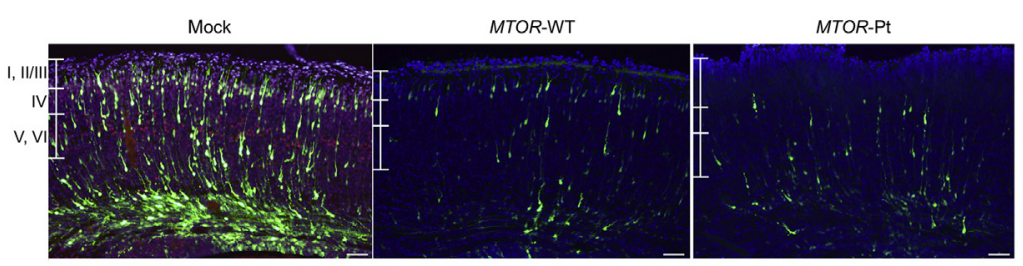

B

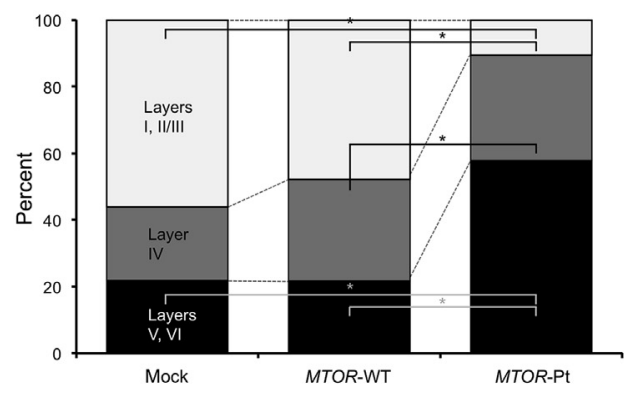

Figure 5 Abnormal distribution of MTOR-transfected cells in the neocortex. A: Many mock cells are located in the layer I and II/III. However, MTOR-Pt cells locate in the deep region. The layer V and VI are confirmed as the localization of Ctip2-labeled cells (red-labeled cells). B: Distribution analysis demonstrates significantly higher ratio of MTOR-Pt cells in the layer V and VI than those of mock and MTOR-WT cells. However, the layer I and II/III exhibits significantly lower ratio of MTOR-Pt cells. Data are expressed as means \pm SEM. $n=3$ MTOR-Pt cells (B); $n=3$ mock cells $(\mathbf{B}) ; n=4$ MTOR-WT (B). ${ }^{\star} P<0.05$. Scale bars $=50 \mu \mathrm{m}$. Ctip2, chicken ovalbumin upstream promoter transcription factor-interacting proteins 2; MTOR-Pt, patient's mutated $m$ TOR transfected cells; MTOR-WT, wild-type mTOR transfected cells. 


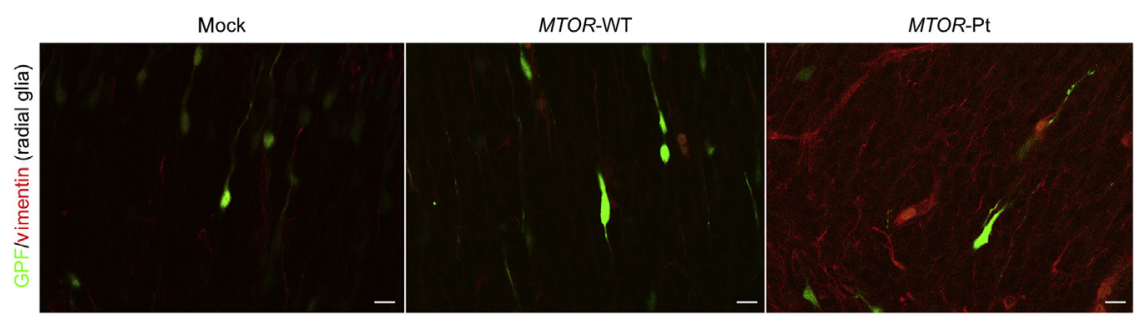

Figure 6 Relation of MTOR-transfected cells and radial glial fibers. Each transfected cell is bipolar in shape and on the radial glial fiber, using vimentin as the marker in the somatosensory cortices. Scale bars $=10 \mu \mathrm{m}$. GFP, green fluorescent protein; MTOR-Pt, patient's mutated $M T O R$ transfected cells; MTOR-WT, wild-type mTOR transfected cells.

activated phosphorylation of MTOR-downstream molecules, and MTOR mutation effect led to more active phosphorylation. These data provide further evidence supporting the HME pathogenicity through MTOR activation identified in the resected dysplastic tissue.

\section{Abnormal Size and Neocortical Distribution in the Mutated MTOR-Transfected Cells}

After in utero electroporation with the vectors at E14.5, we observed the neocortex at E18.5. Mock neocortex showed many transfected cells and well-formed layers. However, wild-type $M T O R$ or MTOR mutation-transfected cells were a few and did not form cortical lamina (Figures 4A and 5). Moreover, MTOR mutation-transfected cells were significantly larger in size than mock or wild-type MTOR (Figure 4B).

We evaluated the MTOR up-regulated cell distribution in the neocortex. It is known that Ctip2-labeled cells are born at E12 to E16 and form the layers V and VI of the neocortex. ${ }^{26}$ We experientially divided three layers based on the Ctip2-labeled cell layer (Figure 5A). As mentioned above, a few mutated MTOR-transfected cells were identified in layers I and II/III but most in the deep layer (Figure 5B). Those results were the same as previous description. $^{11}$

After clarifying whether mutated MTOR-transfected cells were gathering into deep cortical region or intermediate zone, we investigated the relation between cells and the attached radial glial fibers. A radial glial fiber functions in the guidance of a migrating neuron, with attachment and interaction between each other mediated by a complex molecular system. ${ }^{27}$ To understand the pathomechanism forming dysplastic cortex, it is important to know whether MTOR mutant cells can climb on the fiber. Cells transfected with each vector had a bipolar shape and attached on the fiber (Figure 6). Next, we revealed that the hyperactive MTOR worked to induce cell-autonomous changes (Figure 7). To confirm layer formation, we investigated the localization of Foxp1- and Ctip2-labeled cells and the relation with those cells and EGFP-marked cells. It is known that the Foxp1-labeled cells were born after E14.5 and Ctip2-labeled cells were born around E14.5. ${ }^{28,29}$ All Ctip2-labeled cells located to the original position of each transfected neocortex. Foxp1-labeled cells were also distributed diffusely in the cortical plate and intermediate zone. Interestingly, Foxp1-labeled cells with MTOR activity (EGFP-marked cells) might increase in the intermediate zone.

\section{Discussion}

We presented a novel hyperactive $M T O R$ mutation, as a cause of HME pathologic disorder. Already, some genes of the PI3K-Akt-mTOR cell signaling pathway have been known as causative somatic or germline mutations of
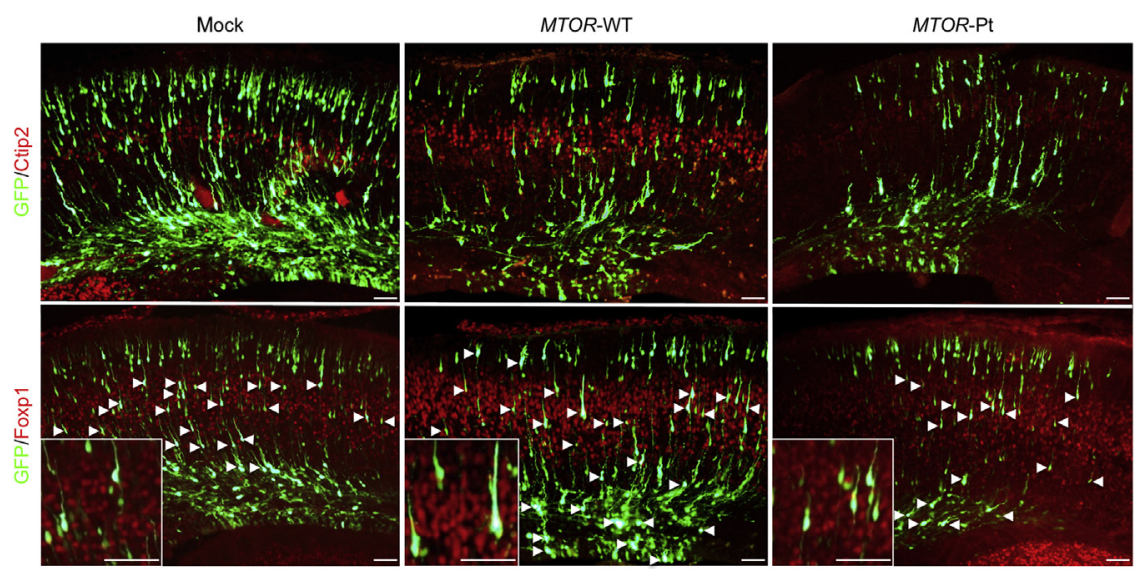

Figure 7 The relation of layer formation and MTOR-transfected cells. Ctip2-labeled cell layer and the forkhead box protein P1 (Foxp1)-labeled cell layer are well observed in each neocortex. There are no merged cells with GFP and Ctip2 in each brain. However, green fluorescent protein (GFP)and Foxp1-merged cells were distributed in the somatosensory cortices (arrowheads). The insets represent the merged cells in the Foxp1-labeled cell layer. Scale bars $=50 \mu \mathrm{m}$. Ctip2, chicken ovalbumin upstream promoter transcription factor-interacting proteins 2; MTOR-Pt, patient's mutated mTOR transfected cells; MTOR-WT, wildtype $m T O R$ transfected cells. 
HME and FCD. ${ }^{10,30}$ As evidence accumulated, the abnormal expression of MTOR and its relative proteins in human pathologic disorder and abnormal positioning in the cortex was described. ${ }^{11,30,31}$ However, how the mutations form cortical dysplasia and result in FCD or HME remains to be understood. Among many MTOR protein functions, we know that it is important in regulating cell growth and various other physiological conditions. ${ }^{27,32}$ Neurodevelopmental diseases are thought to be correlated with hyperactive MTOR, exhibiting neuronal morphologic and neurophysiological abnormalities, and patient's phenotypes of intellectual disability, seizure, autistic behavior, and other neuropsychological problems. ${ }^{24}$ The present study can provide two new pieces of knowledge. First, we revealed that a hyperactive MTORmutated cell climbed on the radial glial fiber in the same pattern of a normal manner ${ }^{33-35}$ but located into the lower area of the cortex or intermediate zone. The fact indicates that in cortical formations derived from MTOR mutation the cell has been normally regulated since the step of the attachment of the radial glial fiber from cell birth, and exhibited a slowed climbing speed. MTOR might not influence the steps, such as cell birth at the subventricular zone, growing multipolar cells to bipolar cells in the intermediate zone, and attachment and interaction of bipolar cells and radial glial fibers as well known. ${ }^{36,37}$ Recently, as our data showed, the phosphoinositide-dependent kinase-1-Akt1 pathway regulates radial neuronal migration, and its dysfunction slows the climbing speed through uncontrolled microtubule formation, but with an as yet unknown relation to MTOR. ${ }^{38}$ MTOR affects the microtubule complex important for neuronal development and modification of synaptic strength. A p70S6K, MTORtargeted molecule is associated with the actin cytoskeleton and colocalized with the Akt1, phosphoinositidedependent kinase-1, and PI3K proteins. Moreover, activated MTOR affects cell migration by p70S6K activation through F-actin domains in cells. ${ }^{39}$ Among many molecules that facilitate the conformation and reconstruction of cell structure, microtubule dynamics may be correlated with phosphorylated S6 and 4EBP1 through MTOR1 complex activation. Second, MTOR hyperactivation might influence cell-autonomous responses. MTORhyperactivated cells demonstrated slow climbing along the radial glial fiber, but the non-MTOR hyperactive Foxp1-labeled cells located the original position of the cortex. The fact suggests that hyperactive MTOR acts by delaying cell migration, disorientation, and overgrowth by itself but never affects the other cells and components. Interestingly, both phenomena were observed with dependence of MTOR active level. We can easily speculate that MTOR hyperactivation completes various morphologic and functional changes within the cell and may lead to dysplastic cortex, featuring the combination of abnormal neuron characteristics and migration and normallooking neurons. From the view of somatic mutations as a cause of HME or FCD, pathologic dysplastic severity can be determined by the MTOR activity level and its expansion by the mutation-yield timing.

\section{Conclusion}

MTOR activity controls cell-autonomous functions such as both cell growth and migration with microtubule/actin complexes in early embryonic brains. It is important for normal cortex formation to receive the restricted limitation of MTOR volume and activity at that time. The disruption can dose dependently lead to cortical dysplasia without running out from the radial glial fiber. Here, we described not only a novel hyperactive MTOR somatic mutation but also the mechanism underlying cell migration delay. The knowledge can provide a major step toward understanding the complex pathophysiology of HME or FCD.

\section{Acknowledgments}

We thank the patient and her family for their participation in this study.

\section{Supplemental Data}

Supplemental material for this article can be found at http://dx.doi.org/10.1016/j.ajpath.2017.01.015.

\section{References}

1. Leventer RJ, Phelan EM, Coleman LT, Kean MJ, Jackson GD, Harvey AS: Clinical and imaging features of cortical malformations in childhood. Neurology 1999, 53:715-722

2. Otsuki T, Honda R, Takahashi A, Kaido T, Kaneko Y, Nakai T, Saito Y, Itoh M, Nakagawa E, Sugai K, Sasaki M: Surgical management of cortical dysplasia in infancy and early childhood. Brain Dev 2013, 35:802-809

3. Crino PB: The mTOR signalling cascade: paving new roads to cure neurological disease. Nat Rev Neurol 2016, 12:379-392

4. Lamouille S, Derynck R: Cell size and invasion in TGF-beta-induced epithelial to mesenchymal transition is regulated by activation of the mTOR pathway. J Cell Biol 2007, 178:437-451

5. Magri L, Cambiaghi M, Cominelli M, Alfaro-Cervello C, Cursi M Pala M, Bulfone A, Garcia-Verdugo JM, Leocani L, Minicucci F, Poliani PL, Galli R: Sustained activation of mTOR pathway in embryonic neural stem cells leads to development of tuberous sclerosis complex-associated lesions. Cell Stem Cell 2011, 9:447-462

6. Lindhurst MJ, Sapp JC, Teer JK, Johnston JJ, Finn EM, Peters K, et al: A mosaic activating mutation in AKT1 associated with the Proteus syndrome. N Engl J Med 2011, 365:611-619

7. Lee JH, Huynh M, Silhavy JL, Kim S, Dixon-Salazar T, Heiberg A Scott E, Bafna V, Hill KJ, Collazo A, Funari V, Russ C, Gabriel SB, Mathern GW, Gleeson JG: De novo somatic mutations in components of the PI3K-AKT3-mTOR pathway cause hemimegalencephaly. Nat Genet 2012, 44:941-945

8. Poduri A, Evrony GD, Cai X, Elhosary PC, Beroukhim R, Lehtinen MK, Hills LB, Heinzen EL, Hill A, Hill RS, Barry BJ, Bourgeois BF, Riviello JJ, Barkovich AJ, Black PM, Ligon KL, 
Walsh CA: Somatic activation of AKT3 causes hemispheric developmental brain malformations. Neuron 2012, 74:41-48

9. Rivière JB, Mirzaa GM, O'Roak BJ, Beddaoui M, Alcantara D, Conway RL, Finding of Rare Disease Genes (FORGE) Canada Consortium, et al: De novo germline and postzygotic mutations in AKT3, PIK3R2 and PIK3CA cause a spectrum of related megalencephaly syndromes. Nat Genet 2012, 44:934-940

10. Jansen LA, Mirzaa GM, Ishak GE, O'Roak BJ, Hiatt JB, Roden WH, Gunter SA, Christian SL, Collins S, Adams C, Rivière JB, St-Onge J, Ojemann JG, Shendure J, Hevner RF, Dobyns WB: PI3K/AKT pathway mutations cause a spectrum of brain malformations from megalencephaly to focal cortical dysplasia. Brain 2015, 138: $1613-1628$

11. Kassai H, Sugaya Y, Noda S, Nakao K, Maeda T, Kano M, Aiba A: Selective activation of mTORC1 signaling recapitulates microcephaly, tuberous sclerosis, and neurodegenerative diseases. Cell Rep 2014, 7: $1626-1639$

12. Gangloff YG, Mueller M, Dann SG, Svoboda P, Sticker M, Spetz JF, Um SH, Brown EJ, Cereghini S, Thomas G, Kozma SC: Disruption of the mouse mTOR gene leads to early postimplantation lethality and prohibits embryonic stem cell development. Mol Cell Biol 2004, 24: 9508-9516

13. Murakami M, Ichisaka T, Maeda M, Oshiro N, Hara K, Edenhofer F, Kiyama H, Yonezawa K, Yamanaka S: mTOR is essential for growth and proliferation in early mouse embryos and embryonic stem cells. Mol Cell Biol 2004, 24:6710-6718

14. Hentges KE, Sirry B, Gingeras AC, Sarbassov D, Sonenberg N, Sabatini D, Peterson AS: FRAP/mTOR is required for proliferation and patterning during embryonic development in the mouse. Proc Natl Acad Sci U S A 2001, 98:13796-13801

15. Cloëtta D, Thomanetz V, Baranek C, Lustenberger RM, Lin S, Oliveri F, Atanasoski S, Rüegg MA: Inactivation of mTORC1 in the developing brain causes microcephaly and affects gliogenesis. J Neurosci 2013, 33:7799-7810

16. Sherman DL, Krols M, Wu LM, Grove M, Nave KA, Gangloff YG, Brophy PJ: Arrest of myelination and reduced axon growth when schwann cells lack mTOR. J Neurosci 2012, 32:1817-1825

17. Arai A, Saito T, Hanai S, Sukigara S, Nabatame S, Otsuki T, Nakagawa E, Takahashi A, Kaneko Y, Kaido T, Saito Y, Sugai K, Sasaki M, Goto Y, Itoh M: Abnormal maturation and differentiation of neocortical neurons in epileptogenic cortical malformation: unique distribution of layer-specific marker cells of focal cortical dysplasia and hemimegalencephaly. Brain Res 2012, 1470:89-97

18. Hanai S, Saito T, Nakagawa E, Arai A, Otsuki T, Sasaki M, Goto Y, Itoh M: Abnormal maturation of non-dysmorphic neurons in focal cortical dysplasia: immunohistochemical considerations. Seizure 2010, 19:274-279

19. Sakakibara T, Sukigara S, Saito T, Otsuki T, Takahashi A, Kaneko Y, Kaido T, Saito Y, Sato N, Kimura Y, Nakagawa E, Sugai K, Sasaki M, Goto Y, Itoh M: Delayed maturation of neurons in focal cortical dysplasia with the transmantle sign: analysis of layer-specific marker expression. J Neuropathol Exp Neurol 2012, 71:741-749

20. Sakakibara T, Sukigara S, Otsuki T, Takahashi A, Kaneko Y, Kaido T, Saito Y, Sato N, Nakagawa E, Sugai K, Sasaki M, Goto Y, Itoh M: Imbalance of interneuron distribution between neocortex and basal ganglia: consideration of epileptogenesis of focal cortical dysplasia. J Neurol Sci 2012, 323:128-133

21. Itoh M, Ide S, Takashima S, Kudo S, Nomura Y, Segawa M, Kubota T, Mori H, Tanaka S, Horie H, Tanabe Y, Goto Y: Methyl CpG-binding protein 2 (a mutation of which causes Rett syndrome) directly regulates insulin-like growth factor binding protein 3 in mouse and human brains. J Neuropathol Exp Neurol 2007, 66:117-123

22. Szczurkowska J, Cwetsch AW, dal Maschio M, Ghezzi D, Ratto GM, Cancedda L: Targeted in vivo genetic manipulation of the mouse or rat brain by in utero electroporation with a triple-electrode probe. Nat Protoc 2016, 11:399-412

23. Shun Z, Silverberg A, Chang CK, Ouyang P: Dunnett's many-to-one test and least square means. J Biopharm Stat 2003, 13:17-28

24. Hardt M, Chantaravisoot N, Tamanoi F: Activating mutations of TOR (target of rapamycin). Genes Cells 2011, 16:141-151

25. Leventer RJ, Scerri T, Marsh AP, Pope K, Gillies G, Maixner W, MacGregor D, Harvey AS, Delatycki MB, Amor DJ, Crino P, Bahlo M, Lockhart PJ: Hemispheric cortical dysplasia secondary to a mosaic somatic mutation in MTOR. Neurology 2015, 84: 2029-2032

26. Brunjes PC, Osterberg SK: Developmental markers expressed in neocortical layers are differentially exhibited in olfactory cortex. PLoS One 2015, 10:e138541

27. Kawauchi T: Cellullar insights into cerebral cortical development: focusing on the locomotion mode of neuronal migration. Front Cell Neurosci 2015, 9:394

28. Ferland RJ, Cherry TJ, Preware PO, Morrisey EE, Walsh CA: Characterization of Foxp2 and Foxp1 mRNA and protein in the developing and mature brain. J Comp Neurol 2003, 460:266-279

29. Leid M, Ishmael JE, Avram D, Shepherd D, Fraulob V, Dollé P: CTIP1 and CTIP2 are differentially expressed during mouse embryogenesis. Gene Expr Patterns 2004, 4:733-739

30. Mirzaa GM, Campbell CD, Solovieff N, Goold CP, Jansen LA, Menon S, et al: Association of MTOR mutations with developmental brain disorders, including megalencephaly, focal cortical dysplasia, and pigmentary mosaicism. JAMA Neurol 2016, 73:836-845

31. Ljungberg MC, Bhattacharjee MB, Lu Y, Armstrong DL, Yoshor D, Swann JW, Sheldon M, D'Arcangelo G: Activation of mammalian target of rapamycin in cytomegalic neurons of human cortical dysplasia. Ann Neurol 2006, 60:420-429

32. Lipton JO, Sahin M: The neurology of mTOR. Neuron 2014, 84: 275-291

33. Noctor SC, Palmer SL, Hasling T, Juliano SL: Interference with the development of early generated neocortex results in disruption of radial glia and abnormal formation of neocortical layers. Cereb Cortex 1999, 9:121-136

34. Noctor SC, Flint AC, Weissman TA, Dammerman RS, Kriegstein AR: Neurons derived from radial glial cells establish radial units in neocortex. Nature 2001, 409:714-720

35. Rakic P: Developmental and evolutionary adaptations of cortical radial glia. Cereb Cortex 2003, 13:541-549

36. Cooper JA: Molecules and mechanisms that regulate multipolar migration in the intermediate zone. Front Cell Neurosci 2014, $8: 386$

37. Pollen AA, Nowakowski TJ, Chen J, Retallack H, Sandoval-Espinosa C, Nicholas CR, Shuga J, Liu SJ, Oldham MC, Diaz A, Lim DA, Leyrat AA, West JA, Kriegstein AR: Molecular identity of human outer radial glia during cortical development. Cell 2015, 163:55-67

38. Itoh Y, Higuchi M, Oishi K, Kishi Y, Okazaki T, Sakai H, Miyata T, Nakajima K, Gotoh Y: PDK1-Akt pathway regulates radial neuronal migration and microtubules in the developing mouse neocortex. Proc Natl Acad Sci U S A 2016, 113:E2955-E2964

39. Berven LA, Willard FS, Crouch MF: Role of the p70(S6K) pathway in regulating the actin cytoskeleton and cell migration. Exp Cell Res 2004, 296:183-195 